\title{
Strong phonon anharmonicity and low thermal conductivity of monolayer tin oxides driven by lone-pair electrons
}

\author{
Wenhui Wan, ${ }^{1}$ Yanfeng Ge, ${ }^{1}$ and Yong Liu ${ }^{1,}$ a) \\ State Key Laboratory of Metastable Materials Science and Technology \& \\ Key Laboratory for Microstructural Material Physics of Hebei Province, \\ School of Science, Yanshan University, Qinhuangdao, 066004, \\ P.R. China
}

Motivated by the excellent electronic and optoelectronic properties of twodimensional (2D) tin oxides, we systematically investigated the thermal conductivity $(\kappa)$ of monolayer $\mathrm{SnO}$ and $\mathrm{SnO}_{2}$ by the first-principles calculations. The room-temperature $\kappa$ of monolayer $\mathrm{SnO}$ and $\mathrm{SnO}_{2}$ reaches $9.6 \mathrm{~W} /(\mathrm{m} \cdot \mathrm{K})$ and 98.8 $\mathrm{W} /(\mathrm{m} \cdot \mathrm{K})$, respectively. The size effect is much weaker for monolayer $\mathrm{SnO}$ than for monolayer $\mathrm{SnO}_{2}$, due to the coexistence of size dependent and independent component in the $\kappa$ of monolayer $\mathrm{SnO}$. The large difference between the $\kappa$ of $2 \mathrm{D}$ tin oxides can be attributed to the small phonon group velocity and strong anharmonicity strength of monolayer SnO. Further electronic structure analysis reveals that the existence of sterically active lone-pair electrons is the key factor for the small $\kappa$ of monolayer SnO. These results provide a guide for the manipulation of thermal transport in the electronic or thermoelectric devices based on 2D tin oxides.

PACS numbers: 63.20.-e 63.22.-m, 68.60.Dv, 63.20.kg

Keywords: Two-dimensional, Tin oxides, Thermal conductivity, lone-pair electrons

Two-dimensional (2D) materials have attracted much attention as the candidates for developing next-generation high-performance electronics, optoelectronics, and spintronic devices. ${ }^{1-3}$ However, prototype $2 \mathrm{D}$ materials such as graphene, transition metal dichalcogenides (TMDs) and phosphorene are usually unstable at high temperature or oxidize gradually in the air. ${ }^{4,5} 2 \mathrm{D}$ metal oxides, by contrast, are environmentally stable, relatively easy to fabricate and typically comprised of non-toxic, naturally occurring elements. ${ }^{6}$ Thus, many efforts have been denoting into the synthesis, fundamental properties and devices applications of metal oxides. ${ }^{7-9}$

Tin oxides are of considerable technological interest as a series of metal oxides. Under atmospheric conditions, tin oxides usually consist of both $\mathrm{SnO}$ and $\mathrm{SnO}_{2} \cdot{ }^{10,11}$ Tin oxides have shown high promise in the field of gas sensing, ${ }^{12,13}$ field effect transistors, ${ }^{14,15}$ as anode materials, ${ }^{16,17}$ thermoelectrics ${ }^{9}$ and optoelectronic devices. ${ }^{18}$ Besides, $\mathrm{SnO}$ can exhibit bipolar conductivity under suitable conditions which is of great potential in the design of p-n junction. ${ }^{19}$ Meanwhile, transition metal doped $\mathrm{SnO}_{2}$ was identified as dilute magnetic semiconductors with curie temperature $T_{c}$ close to $650 \mathrm{~K} .{ }^{20} 2 \mathrm{D}$ materials usually possess unique properties that differ from their bulk counterparts. The optical and electrical properties of $\mathrm{SnO}$ nanosheets were demonstrated to be strongly dependent on its dimensions. ${ }^{21}$ Bulk $\mathrm{SnO}$ is a layered material with a tetragonal PbO-type crystallographic structure. Recently, monolayer $\mathrm{SnO}$ has been synthesized. ${ }^{6,22}$ Ferromagnetism was predicted in the hole-doped monolayer $\mathrm{SnO}$, due to its valence band which has a Mexican-hat band edge. ${ }^{23}$ On the other side, though bulk $\mathrm{SnO}_{2}$ has a rutile crystal structure, porous monolayer $\mathrm{SnO}_{2}$ nanofilm has been fabricated, ${ }^{24}$ indicating that the growth of $2 \mathrm{D} \mathrm{SnO}_{2}$ is likely to be realized in near future. A T-phase structure was predicted as the ground-state structure of monolayer $\mathrm{SnO}_{2} \cdot{ }^{25}$ The magnetic properties of Co-doped and transition metal doped monolayer $\mathrm{SnO}_{2}$ have been investigated. ${ }^{26,27}$ Compared to the intense research on the synthesis and electronic properties of $2 \mathrm{D}$ tin oxides, their thermal properties receive less attention. Thermal conductivity $(\kappa)$ is a fundamental physical quantity which has a large influence on many

a)Electronic mail: ycliu@ysu.edu.cn or yongliu@ysu.edu.cn 

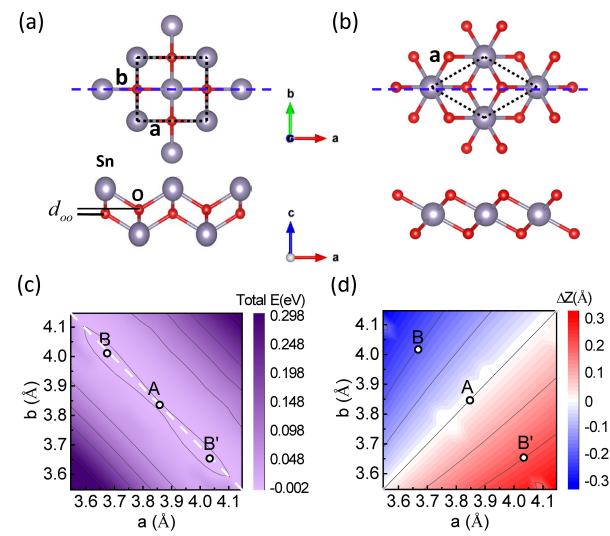

FIG. 1. A side view and a top view of crystal structure of (a) monolayer $\mathrm{SnO}$ and (b) $\mathrm{SnO}_{2}$ with the bash line showing the unit cell. (c) The free-energy contour of $\mathrm{SnO}$ as function of lattice constant $a$ and $b$. (d) The distance between $\mathrm{O}$ atoms $d_{o o}$ along the out-of-plane direction as a function of $a$ and $b$.

applications such as the heat dissipation of the integrated electronic devices and the efficiency of thermoelectric conversion. ${ }^{28,29}$ The thermal transport properties of $2 \mathrm{D}$ tin oxides have not been measured in experiment up to date and call for a systematical investigation at a microscopic level.

In this work, we calculated the thermal conductivity of monolayer $\mathrm{SnO}$ and $\mathrm{SnO}_{2}$ by the first-principles calculations coupled with the phonon Boltzmann transport equation (BTE). The stable structures of $2 \mathrm{D}$ tin oxides are identified. Monolayer $\mathrm{SnO}_{2}$ have a high roomtemperature $\kappa$ which is dominated by acoustic phonons. In contrast, monolayer $\mathrm{SnO}$ has a $\kappa$ which is almost ten times lower than that of $\mathrm{SnO}_{2}$. The size effect of $\kappa$ in tin oxides was investigated. We analyzed the role of phonon group velocity, anharmonicity and phase space for anharmonicity scattering in the thermal transport. The imparity of $\kappa$ between 2D tin oxides can be attributed to the lone-pair electrons in the monolayer $\mathrm{SnO}$.

The lattice thermal conductivity $\kappa$ is obtained by ${ }^{30}$

$$
\kappa_{\alpha \beta}=\frac{1}{N \Omega} \sum_{\mathbf{q}, s} C_{\mathbf{q}, s} v_{\mathbf{q}, s}^{\alpha} v_{\mathbf{q}, s}^{\beta} \tau_{\mathbf{q}, s},
$$

where $N$ and $\Omega$ are the number of $\mathbf{q}$ point and volume of the unit cell, respectively. $C_{\mathbf{q}, s}$, $v_{\mathbf{q}, s}^{\alpha}$ and $\tau_{\mathbf{q}, s}$ is the mode specific capacity, group velocity along the $\alpha$-th direction and phonon lifetime in the single-mode relaxation time approximation (RTA) of the phonon with wavevector $\mathbf{q}$ and branch index $s$, respectively. $\tau_{\mathbf{q}, s}$ was obtained by combing the anharmonic scattering, isotopic impurities scattering and boundary scattering, according to the Matthiessen rule ${ }^{30}$

$$
\frac{1}{\tau_{\mathbf{q}, s}}=\frac{1}{\tau_{\mathbf{q}, s}^{a n}}+\frac{1}{\tau_{\mathbf{q}, s}^{i s o}}+\frac{1}{\tau_{\mathbf{q}, s}^{b}}
$$

where the completely rough boundary with size of $L$ was used, leading to $1 / \tau_{\mathbf{q}, s}^{b}=L /\left|v_{\mathbf{q}, s}\right|$. All computational details are given in the supplementary material

Figure 1(a) displays the lattice crystal of monolayer $\mathrm{SnO}$, with two $\mathrm{Sn}$ and $\mathrm{O}$ atoms in a unit cell. $a$ and $b$ is the lattice constant along $x$ - and $y$-axis, respectively. Unlike bulk and bilayer $\mathrm{SnO}$, monolayer $\mathrm{SnO}$ with $a=b=3.847 \AA$ (labeled as phase A) is unstable (see Fig. $\mathrm{S} 2(\mathrm{a}-\mathrm{c})$ in the supplementary material), agreeing with previous works. ${ }^{31,32}$ The eigenvectors of phonon modes with imaginary frequencies correspond to the anti-directional movement of $\mathrm{O}$ atoms along the $z$-axis. The contour of total energy of monolayer $\mathrm{SnO}$, as the function of $a$ and $b$, is presented in Fig. 1(c). Compared to phase A, another rectangle structure is more stable which has $a=4.022 \AA$ and $b=3.663 \AA$, or vice versa (labeled as phase $\mathrm{B}$ or 

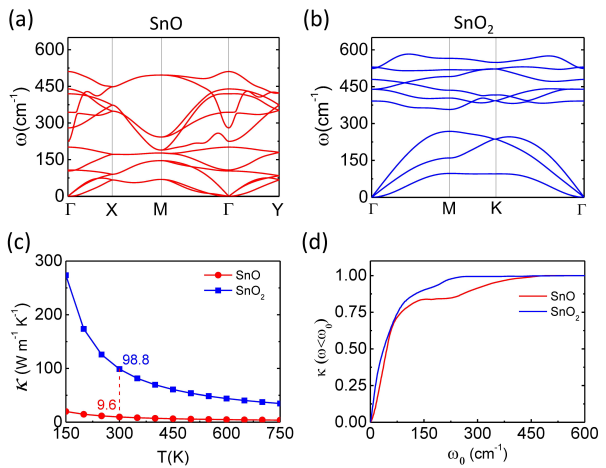

FIG. 2. The phonon dispersion of (a) monolayer $\mathrm{SnO}$ and (b) monolayer $\mathrm{SnO}_{2}$. (c) Temperature dependence of $\kappa$ of monolayer $\mathrm{SnO}$ and monolayer $\mathrm{SnO}_{2}$. (d) At room temperature, the frequency dependence of the normalized cumulative $\kappa\left(\omega<\omega_{0}\right)$ for monolayer $\mathrm{SnO}$ (red) and $\mathrm{SnO}_{2}$ (blue).

$\left.\mathrm{B}^{\prime}\right)$. For more clarity, Fig. S2(d) in the supplementary material displays the energy profile along the path B-A-B' (see bash line in Fig. 1(c)). Meanwhile, the distance between $\mathrm{O}$ atoms $\left(d_{o o}\right)$ along the $z$ direction changes from zero in phase $\mathrm{A}$ to $0.2 \AA$ in phase $\mathrm{B}$ (see Fig. 1(d)). That is consistent with aforementioned eigen-displacement of the imaginary modes in phase A. Moreover, the structural instability of phase A was further identified with different simulation methods (see Fig. S2(d) in the supplementary material). For the case of monolayer $\mathrm{SnO}_{2}$, we adopted T-phase structure ${ }^{25}$ (see Fig. 1(b)). The thickness of monolayer $\mathrm{SnO}_{2}$ is chosen by the interlayer distance of bilayer $\mathrm{SnO}_{2}$ with the most stable structure (see Fig. S3 in the supplementary material). All the optimized structural parameters are listed in Table. I. The crystal symmetry of crystal lattice of monolayer SnO is $D_{2 h}$ while that of monolayer $\mathrm{SnO}_{2}$ is $D_{3 d}$ which contains an inversion center on Sn site. The bonding length of $\mathrm{Sn}-\mathrm{O}$ is longer in monolayer $\mathrm{SnO}$ than that in monolayer $\mathrm{SnO}_{2}$.

Pristine 2D tin oxides are non-magnetic semiconductors (see Fig. S4 in the supplementary material) and heat is mainly carried by phonons. The phonon dispersions of monolayer $\mathrm{SnO}$ in phase $\mathrm{B}$ and monolayer $\mathrm{SnO}_{2}$ are displayed in Fig. 2(a,b), which ensure their structural stability. The lowest three branches are the out-of-plane acoustic (ZA), transverse acoustic (TA) and longitudinal acoustic (LA) branch. ZA branch has a quadratic dispersion near the $\Gamma$ point. ${ }^{33}$ An acoustic-optical gap is absent and present in the phonon dispersion of monolayer $\mathrm{SnO}$ and $\mathrm{SnO}_{2}$, respectively. The low- and high-frequency phonon modes are mainly dominated by the vibration of $\mathrm{Sn}$ and $\mathrm{O}$ atoms, respectively (see Fig. S5 in the supplementary material).

The temperatures (T) dependence of $\kappa$ without boundary scattering is shown in Fig. 2(c). At room temperature, the $\kappa$ of monolayer $\mathrm{SnO}_{2}$ reaches $98.8 \mathrm{~W} /(\mathrm{m} \cdot \mathrm{K})$, comparable with that of conventional semiconducting Ge $(65 \mathrm{~W} /(\mathrm{m} \cdot \mathrm{K}))^{34}$ and $\mathrm{GaAs}(45 \mathrm{~W} /(\mathrm{m} \cdot \mathrm{K})) \cdot{ }^{34}$ Though the lattice crystal of monolayer $\mathrm{SnO}$ is anisotropic, its $\kappa$ is almost isotopic. The small anisotropy of $\kappa$ is keeping pace with that of phonon group velocity, ${ }^{35}$ which can be further attributed to the similar atomic bonding characteristics along the $x$ - and $y$-direction (see Fig. S6 in the supplementary material). It is possible to induce the anisotropic thermal transport into $2 \mathrm{D}$ tin oxides by dislocation ${ }^{36}$ or uniaxial strain ${ }^{37}$ for broadening their

TABLE I. The lattice constant $(a, b)$, Sn-O bonding length $\left(d_{S n-O}\right)$ and thickness $(l)$ of monolayer tin oxides.

\begin{tabular}{lccc}
\hline \hline & $a, b(\AA)$ & $d_{\text {SnO }}(\AA)$ & $l(\AA)$ \\
\hline $\mathrm{SnO}$ & $4.022,3.663$ & $2.281,2.228$ & 4.826 \\
$\mathrm{SnO}_{2}$ & 3.227 & 2.121 & 4.272 \\
\hline \hline
\end{tabular}



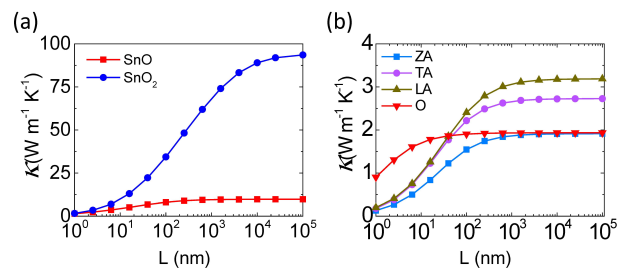

FIG. 3. (a) The lateral size $(L)$ dependence of the $\kappa$ of monolayer $\mathrm{SnO}$ and $\mathrm{SnO}_{2}$. (b) The $L$ dependence of the $\kappa$ contributed by different branches in monolayer $\mathrm{SnO}$.

potential applications in future. We will only discuss the $\kappa$ along the $x$-axis hereafter. The room-temperature $\kappa$ of monolayer $\mathrm{SnO}$, however, is only $9.6 \mathrm{~W} /(\mathrm{m} \cdot \mathrm{K})$ which is ten times smaller than that of $\mathrm{SnO}_{2}$. Such a large imparity of $\kappa$ is consistent with the large difference between the $\kappa$ of bulk $\mathrm{SnO}$ and $\mathrm{SnO}_{2}$ observed in experiment. ${ }^{38}$ Therefore, thermal transport is the limit factor in electronic applications of $2 \mathrm{D} \mathrm{SnO}$ rather than $2 \mathrm{D} \mathrm{SnO}_{2}$.

By fitting the phonon density of states (DOS) at low phonon frequencies with a Debye model, ${ }^{39}$ we obtained Debye temperature $\theta_{D}$ as $259 \mathrm{~K}$ and $356 \mathrm{~K}$ for monolayer $\mathrm{SnO}$ and $\mathrm{SnO}_{2}$, respectively. As a result, the $T$ dependence of $\kappa$ of monolayer $\mathrm{SnO}_{2}$ follows the relation of $\kappa \sim e^{-\theta_{D} / \beta T}$ at temperature lower than $\theta_{D} \cdot{ }^{40}$ Here $\beta$ is an empirical parameter which was fitted as 1.02 (see Fig. S7(d) in the supplementary material). At temperature substantially beyond $\theta_{D}$, the overall $T$ dependence of $\kappa$ of $2 \mathrm{D}$ tin oxides restores the $1 / T$ behavior (see Fig. S7 in the supplementary material).

We found that the effect of isotopic impurities scattering to $\kappa$ is small compared with the anharmonic scattering in the temperature above $250 \mathrm{~K}$. For example, if we exclude the $1 / \tau_{\mathbf{q}, s}^{i s o}$ from the total $1 / \tau_{\mathbf{q}, s}$ in Eq. 2, the room-temperature $\kappa$ will increase only by $0.6 \%$ and $3.3 \%$ for monolayer $\mathrm{SnO}$ and $\mathrm{SnO}_{2}$, respectively. The $1 / \tau_{\mathbf{q}, s}^{i s o}$ is inversely proportional to atomic mass ${ }^{30}$ which is not very small for tin oxides.

The normalized cumulative $\kappa\left(\omega<\omega_{0}\right)$, which represents the contribution of $\kappa$ from the phonons with frequencies less than $\omega_{0}$, is shown in Fig. 2(d). In the case of monolayer $\mathrm{SnO}$, apart from the acoustic phonons, optical phonons with frequency up to $470 \mathrm{~cm}^{-1}$ have a non-negligible contribution $(\sim 20 \%)$ to $\kappa$ (see Fig. 2(d) and Fig. S8(a) in the supplementary material). That can be attributed to the high dispersive relation and large group velocity of optical phonons (see Fig. 2(a) and Fig. 4(a)). In contrast, the $\kappa$ of monolayer $\mathrm{SnO}_{2}$ is dominated by acoustic phonons with frequency less than $250 \mathrm{~cm}^{-1}$ (see Fig. 2(d)).

The phonon scattering from rough edge can decrease the $\kappa$ of semiconductors to achieve excellent thermoelectricity. ${ }^{29}$ Here the size effect of $\kappa$ is discussed in the range of diffusive thermal transport. The lateral size $(L)$ dependence of room-temperature $\kappa$ and the contribution of phonons from different branches are displayed in Fig. 3. At room temperature, the mean free path (MFP) of phonon is about $10^{3}$ and $10^{5} \mathrm{~nm}$ for monolayer $\mathrm{SnO}$ and $\mathrm{SnO}_{2}$, respectively. In the case of monolayer $\mathrm{SnO}$, though the contribution of $\kappa$ from acoustic phonons can be decreased by enhanced boundary scattering, the contribution from optical phonons is almost size independent until the $L$ decreases down to $10 \mathrm{~nm}$ (see Fig. 3(b)), due to its small MFP. Thus, as the lateral size of $2 \mathrm{D} \mathrm{SnO}$ decreases from $10^{5} \mathrm{~nm}$, nanostructuring might not be an efficient method to reduce its $\kappa$, due to the coexistence of size dependent and independent component. In contrast, the $\kappa$ of $2 \mathrm{D} \mathrm{SnO}_{2}$, which is dominated by acoustic phonons, can be effectively decreased by edge roughness scattering (see Fig. 3(a)).

The large difference between the $\kappa$ of monolayer $\mathrm{SnO}$ and $\mathrm{SnO}_{2}$ can not be simply attributed to the different mean atomic mass. We analyzed the role of each term in Eq. 1 in determining the $\kappa$ of 2D tin oxides. Figure 4(a) displays the frequency dependence of mode group velocity $v_{\mathbf{q}, s}$. The $v_{\mathbf{q}, s}$ of low-frequency phonon modes $\left(\omega<170 \mathrm{~cm}^{-1}\right)$ in monolayer $\mathrm{SnO}_{2}$ is higher than that in monolayer $\mathrm{SnO}$. The group velocity of phonon is large for materials with light atomic mass and strong bonding. The bond strength can be reflected by the $2 \mathrm{D}$ elastic module $C_{2 \mathrm{D}}$, which can be obtained through a fitting process of total energy 

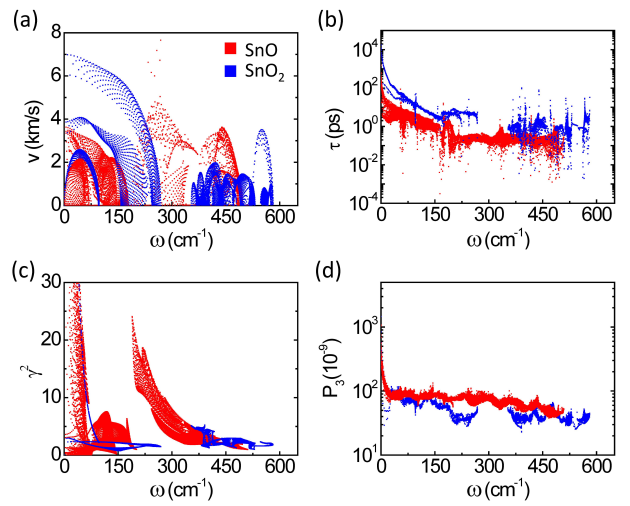

FIG. 4. At room temperature, (a) mode group velocity $v_{\mathbf{q}, s}$, (b) mode phonon life time $\tau_{\mathbf{q}, s}$, (c) The square of mode grüneisen parameter $\gamma_{\mathbf{q}, s}^{2}$ and (d) phase space for anharmonic scattering $P_{3 \mathbf{q}, s}$ for monolayer $\mathrm{SnO}$ (red) and $\mathrm{SnO}_{2}$ (blue), respectively.

with respect to strain. ${ }^{41}$ The $C_{2 \mathrm{D}}$ of monolayer $\mathrm{SnO}$ along $x$ - and $y$-axis is $40.96 \mathrm{~J} / \mathrm{m}^{2}$ and $34.98 \mathrm{~J} / \mathrm{m}^{2}$, much smaller than $C_{2 \mathrm{D}}=260.53 \mathrm{~J} / \mathrm{m}^{2}$ of monolayer $\mathrm{SnO}_{2}$ (see Fig. S9 in the supplementary material). The weak $\mathrm{Sn}-\mathrm{O}$ bonding results in small phonon group velocity as well as aforementioned long Sn-O bond length in monolayer SnO (see Table. I).

The mode phonon lifetimes $\tau_{\mathbf{q}, s}$ of $2 \mathrm{D}$ tin oxides at room temperature are shown in Fig. 4(b). It can be seen that the overall $\tau_{\mathbf{q}, s}$ of monolayer $\mathrm{SnO}_{2}$ is larger than that of monolayer SnO. As mentioned above, anharmonic effect dominates the phonon scattering in the thermal transport of $2 \mathrm{D}$ tin oxides. The scattering rate $1 / \tau_{\mathbf{q}, s}^{a n}$ depends on both the square of Grüneisen parameter $\gamma_{\mathbf{q}, s}^{2}$ and the available three-phonon phase space $\left(\mathrm{P}_{3}\right)_{\mathbf{q}, s} \cdot{ }^{40}$ The former one describes the strength of anharmonicity. The latter one represents the number of anharmonic scattering channel for phonon absorption processes and emission processes, according to the energy and momentum conservations. ${ }^{30}$

The overall $\gamma_{\mathbf{q}, s}^{2}$ of monolayer $\mathrm{SnO}$ is much larger than that of monolayer $\mathrm{SnO}_{2}$, which can be seen from Fig. 4(c). That is also consistent with the fact that the acoustic phonon branches of monolayer $\mathrm{SnO}$ are significantly softened compared to that of monolayer $\mathrm{SnO}_{2}$ (see Fig. 2). The overall $\left(P_{3}\right)_{\mathbf{q}, s}$ of monolayer $\mathrm{SnO}$ is slightly larger than that of $\mathrm{SnO}_{2}$ (see Fig. 4(d)). In the phonon dispersion of monolayer $\mathrm{SnO}$, the soft acoustic phonons and intersection between acoustic and optical branches (see Fig. 2(a)) provide more channels for anharmonic scattering, especially for emission process of high-frequency phonons (see Fig. S10 in the supplementary material). Combining with Fig. 4(c) and 4(d), the small $\tau_{\mathbf{q}, s}$ in monolayer $\mathrm{SnO}$ is mainly due to the large $\gamma_{\mathbf{q}, s}^{2}$. Thus, the much small $\kappa$ of monolayer $\mathrm{SnO}$ arises from the small phonon group velocity and strong anharmonicity strength.

The large imparity between the anharmonicity strength of tin oxides can be further attributed to the electron configuration. The projected density of states (PDOS) of tin oxides are shown in Fig. 5(a) and 5(b). In the valence band of monolayer SnO, Sn-5s electrons interact with the O- $2 p$ states, with the bonding states locating between -8 and $-5 \mathrm{eV}$ and anti-bonding states appears at upper valence bands (see Fig. 5(a) and Fig. S11 in the supplementary material). The anti-bonding states, with a large contribution from the Sn- $5 s$ states, can hybridize with Sn-5p states due to the lack of inversion symmetry. This sort of hybridization shifts the energy of anti-bonding states downward, thereby stabilizing it at the top of valence bands. ${ }^{42}$ The filled anti-bonding states result in the sterically active lone-pair electrons (see Fig. S11 in the supplementary material), similar to the case of bulk $\mathrm{SnO} .^{43}$ The electronic local function (ELF) was calculated with the location of cross-section indicated by the blue line in Fig. 1(a, b), which can directly give a picture of lone-pair electrons. The ELF shows a strong electron localization around the Sn atom (see Fig. 5(c)), consistent with the distribution of lone pair at Sn. As a result, the overlapping wave functions of the lone-pair electrons and nearby $\mathrm{O}-2 p$ bonding electrons will induce a nonlinear 

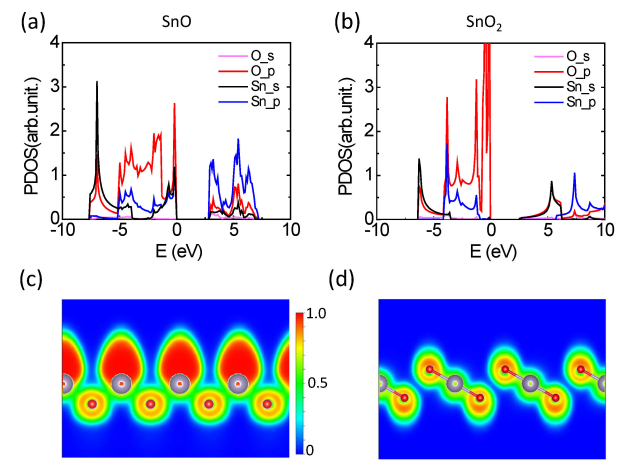

(d)

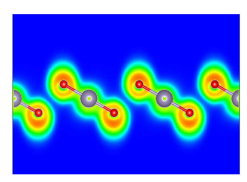

FIG. 5. (a, b) are projected density of states of monolayer (a) $\mathrm{SnO}$ and (b) $\mathrm{SnO}_{2}$, respectively. The electronic local function of monolayer (c) $\mathrm{SnO}$ and (d) $\mathrm{SnO}_{2}$.

repulsive electrostatic force during thermal agitation, ${ }^{44,45}$ leading to enhanced anharmonicity and small phonon lifetime. Additionally, it is indicated that chemical functionalization can effectively modulate the $\kappa$ of $2 \mathrm{D} \mathrm{SnO}$ since the lone-pair electrons distribute on the surface.

In contrast to $\mathrm{SnO}$, the lattice crystal of monolayer $\mathrm{SnO}_{2}$ is centrosymmetric. The mixing between Sn-5p states and anti-bonding states of $\mathrm{O}-2 p$ and $\mathrm{Sn}-5 s$ is prohibited due to the different parity of $s$ and $p$ orbital. The anti-bonding states of O- $2 p$ and Sn- $5 s$ is above the Fermi level and unfilled. Thus, both the $s$ and $p$ electrons of Sn atom participate in Sn-O bonding in a form of $s p^{3}$ hybridization. Electrons transfer from Sn to O atom, as shown in Fig. 5(d) and Fig. S11. The absence of lone-pair electrons make anharmonicity strength to be smaller for monolayer $\mathrm{SnO}_{2}$ than for monolayer $\mathrm{SnO}$ (see Fig. 4(c)), resulting in longer phonon life time (see Fig. 4(b)).

In conclusion, based on the first-principles calculations, we calculated the thermal conductivity of monolayer $\mathrm{SnO}$ and $\mathrm{SnO}_{2}$. The tetragonal lattice of monolayer $\mathrm{SnO}$ spontaneously transforms to a rectangle one. The room-temperature $\kappa$ of monolayer $\mathrm{SnO}$ and $\mathrm{SnO}_{2}$ arrives at $9.6 \mathrm{~W} /(\mathrm{m} \cdot \mathrm{K})$ and $98.8 \mathrm{~W} /(\mathrm{m} \cdot \mathrm{K})$, respectively. The size effect of $\kappa$ is much weaker for monolayer $\mathrm{SnO}$ than for monolayer $\mathrm{SnO}_{2}$, due to the coexistence of size-dependent and size-independent contribution of $\kappa$ in monolayer $\mathrm{SnO}$. The small $\kappa$ of monolayer $\mathrm{SnO}$ is due to the small phonon group velocity and strong anharmonicity strength. That can be further attributed to the existence of sterically active lone-pair electrons distributed on the surface of monolayer SnO. This work may give a guide for the thermal management in the electronic or thermoelectrical devices based on 2D tin oxides.

\section{SUPPLEMENTARY MATERIAL}

See supplementary material for the computational details; The convergence test of $\kappa$; The phonon dispersion of monolayer, bilayer and bulk $\mathrm{SnO}$ with $\mathrm{a}=\mathrm{b}$; The structural and energetical properties of bilayer $\mathrm{SnO}_{2}$; The 2D effective elastic module, band structure, projected phonon density of states of monolayer $\mathrm{SnO}$ and $\mathrm{SnO}_{2}$; The isotropic $\kappa$ of monolayer $\mathrm{SnO}$; The temperature dependence of $\kappa$ of monolayer $\mathrm{SnO}$ and $\mathrm{SnO}_{2}$; The contribution of $\kappa$ from different branches; The three-phonon scattering phase space $\left(P_{3}\right)_{\mathbf{q}, s}$ for absorption and emission process; The distribution of charge density of monolayer $\mathrm{SnO}$ and $\mathrm{SnO}_{2}$. 


\section{ACKNOWLEDGMENTS}

This work was supported by the NSFC (Grants No.11747054), the Specialized Research Fund for the Doctoral Program of Higher Education of China (Grant No.2018M631760), the Project of Heibei Educational Department, China (No. ZD2018015 and QN2018012), and the Advanced Postdoctoral Programs of Hebei Province (No.B2017003004).

${ }^{1}$ K. S. Novoselov, A. K. Geim, S. V. Morozov, D. Jiang, M. I. Katsnelson, I. V. Grigorieva, S. V. Dubonos, and A. A. Firsov, Nature 438, 197 (2005).

${ }^{2}$ M. Chhowalla, H. S. Shin, G. Eda, L.-J. Li, K. P. Loh, and H. Zhang, Nat. Chem. 5, 263 (2013).

${ }^{3}$ V. Nicolosi, M. Chhowalla, M. G. Kanatzidis, M. S. Strano, and J. N. Coleman, Science 340, 1226419 (2013).

${ }^{4}$ A. Ziletti, A. Carvalho, D. K. Campbell, D. F. Coker, and A. H. Castro Neto, Phys. Rev. Lett. 114, 046801 (2015).

${ }^{5}$ S. Cui, Z. Wen, X. Huang, J. Chang, and J. Chen, Small 11, 2305-2313 (2015).

${ }^{6}$ T. Daeneke, P. Atkin, R. Orrell-Trigg, A. Zavabeti, T. Ahmed, S. Walia, M. Liu, Y. Tachibana, M. Javaid, A. D. Greentree, S. P. Russo, R. B. Kaner, and K. Kalantar-Zadeh, ACS Nano 11, 10974-10983 (2017).

${ }^{7}$ R. Sui and P. Charpentier, Chem. Rev. 112, 3057-3082 (2012).

${ }^{8}$ Y. Tokura and N. Nagaosa, Science 288, 462-468 (2000).

${ }^{9}$ S. A. Miller, P. Gorai, U. Aydemir, T. O. Mason, V. Stevanovi, E. S. Toberer, and G. J. Snyder, J. Mater. Chem. C 5, 8854-8861 (2017).

${ }^{10}$ P. D. Padova, M. Fanfoni, R. Larciprete, M. Mangiantini, S. Priori, and P. Perfetti, Surf. Sci. 313, 379 - 391 (1994).

${ }^{11}$ J.-H. Lee, M. Yoo, D. Kang, H.-M. Lee, W.-h. Choi, J. W. Park, Y. Yi, H. Y. Kim, and J.-S. Park, ACS Appl. Mater. Interfaces 10, 33335-33342 (2018).

${ }^{12}$ V. X. Hien, J.-H. Lee, J.-J. Kim, and Y.-W. Heo, Sens. Actuators, B 194, $134-141$ (2014).

${ }^{13}$ V. Kumar, S. Sen, K. Muthe, N. Gaur, S. Gupta, and J. Yakhmi, Sens. Actuators, B 138, $587-590$ (2009).

${ }^{14}$ L. Qiang, W. Liu, Y. Pei, G. Wang, and R. Yao, Solid-State Electron. 129, 163 - 167 (2017).

${ }^{15}$ E. N. Dattoli, Q. Wan, W. Guo, Y. Chen, X. Pan, and W. Lu, Nano Lett. 7, 2463-2469 (2007).

${ }^{16}$ F. Zhang, J. Zhu, D. Zhang, U. Schwingenschlgl, and H. N. Alshareef, Nano Lett. 17, 1302-1311 (2017).

${ }^{17}$ L. Yuan, Z. Guo, K. Konstantinov, H. Liu, and S. Dou, J. Power Sources 159, $345-348$ (2006).

${ }^{18}$ F.-S. Tsai, S.-J. Wang, Y.-C. Tu, Y.-W. Hsu, C.-Y. Kuo, Z.-S. Lin, and R.-M. Ko, Appl. Phys Express 4, 025002 (2011).

${ }^{19}$ K. Nomura, T. Kamiya, and H. Hosono, Adv. Mater. 23, 3431-3434 (2011).

${ }^{20}$ S. B. Ogale, R. J. Choudhary, J. P. Buban, S. E. Lofland, S. R. Shinde, S. N. Kale, V. N. Kulkarni, J. Higgins, C. Lanci, J. R. Simpson, N. D. Browning, S. Das Sarma, H. D. Drew, R. L. Greene, and T. Venkatesan, Phys. Rev. Lett. 91, 077205 (2003).

${ }^{21}$ M. Singh, E. D. Gaspera, T. Ahmed, S. Walia, R. Ramanathan, J. van Embden, E. Mayes, and V. Bansal, 2D Materials 4, 025110 (2017).

${ }^{22}$ K. J. Saji, K. Tian, M. Snure, and A. Tiwari, Adv. Electron. Mater. 2, 1500453 (2016).

${ }^{23}$ L. Seixas, A. S. Rodin, A. Carvalho, and A. H. Castro Neto, Phys. Rev. Lett. 116, 206803 (2016).

${ }^{24}$ H. Chen, L. Hu, X. Fang, and L. Wu, Adv. Funct. Mater. 22, 1229-1235 (2012).

${ }^{25}$ W.-Z. Xiao, G. Xiao, and L.-L. Wang, J. Chem. Phys. 145, 174702 (2016).

${ }^{26}$ H.-X. Luan, C.-W. Zhang, F. Li, P. Li, M.-J. Ren, M. Yuan, W.-X. Ji, and P.-J. Wang, RSC Adv. 4, 9602-9607 (2014).

${ }^{27}$ Y. Feng, W.-X. Ji, B.-J. Huang, X.-l. Chen, F. Li, P. Li, C.-w. Zhang, and P.-J. Wang, RSC Adv. 5, $24306-24312(2015)$

${ }^{28}$ J. Zou and A. Balandin, J. Appl. Phys. 89, 2932-2938 (2001).

${ }^{29}$ A. I. Hochbaum, R. Chen, R. D. Delgado, W. Liang, E. C. Garnett, M. Najarian, A. Majumdar, and P. Yang, Nature 451, 163-167 (2008).

${ }^{30}$ W. Li, J. Carrete, N. A. Katcho, and N. Mingo, Comput. Phys. Commun. 185, $1747-1758$ (2014).

${ }^{31}$ W. Zhou and N. Umezawa, Phys. Chem. Chem. Phys. 17, 17816-17820 (2015).

${ }^{32}$ N. Mounet, M. Gibertini, P. Schwaller, D. Campi, A. Merkys, A. Marrazzo, T. Sohier, I. E. Castelli, A. Cepellotti, G. Pizzi, and N. Marzari, Nat. Nanotechnol. 13, 246-252 (2018).

${ }^{33}$ J. Carrete, W. Li, L. Lindsay, D. A. Broido, L. J. Gallego, and N. Mingo, Mater. Res. Lett. 4, $204-211$ (2016).

${ }^{34}$ D. T. Morelli and S. G. A., edited by S. L. Shindé and J. Goela (Springer-Verlag New York, 2006).

${ }^{35}$ G. Qin, Z. Qin, W.-Z. Fang, L.-C. Zhang, S.-Y. Yue, Q.-B. Yan, M. Hu, and G. Su, Nanoscale 8, 11306-11319 (2016).

${ }^{36}$ B. Sun, G. Haunschild, C. Polanco, J. Z.-J. Ju, L. Lindsay, G. KoblmMei ller, and Y. K. Koh, Nat. Mater. (2018), 10.1038/s41563-018-0250-y

${ }^{37}$ D. Fan, H. Sigg, R. Spolenak, and Y. Ekinci, Phys. Rev. B 96, 115307 (2017).

${ }^{38}$ S. Kuwahara, S. at Tanusilp, Y. Ohishi, H. Muta, S. Yamanaka, and K. Kurosaki, Mater. Trans. 59, 1022-1029 (2018).

${ }^{39}$ A. Togo and I. Tanaka, Scripta Mater. 108, $1-5$ (2015). 
${ }^{40}$ J. M. Ziman, International series of monographs on physics (Clarendon Press, Oxford, 1960).

${ }^{41}$ J. Qiao, X. Kong, Z.-X. Hu, F. Yang, and W. Ji, Nat. Commun. 5, 4475 (2014).

${ }^{42}$ G. W. Watson and S. C. Parker, J. Phys. Chem. B 103, 1258-1262 (1999).

${ }^{43}$ G. W. Watson, J. Chem. Phys. 114, 758-763 (2001).

${ }^{44}$ E. J. Skoug and D. T. Morelli, Phys. Rev. Lett. 107, 235901 (2011).

${ }^{45}$ G. Qin, Z. Qin, H. Wang, and M. Hu, Nano Energy 50, 425 - 430 (2018). 